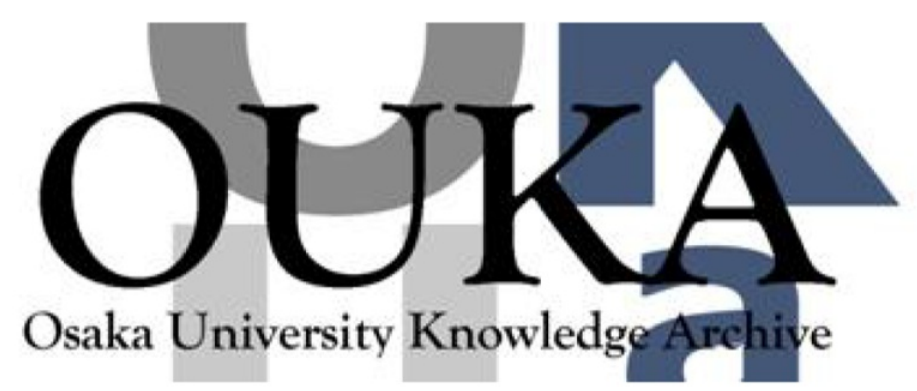

\begin{tabular}{|c|l|}
\hline Title & $\begin{array}{l}\text { Fast Ion Generation in Ultra-Intense Laser } \\
\text { Interactions with Plasmas }\end{array}$ \\
\hline Author(s) & Habara, H.; Kodama, R.; Sentoku, Y. et al. \\
\hline Citation & $\begin{array}{l}\text { Proceedings of SPIE - The International Society } \\
\text { for Optical Eng ineering. 3886 p.513-p. 520 }\end{array}$ \\
\hline Issue Date & 2000 \\
\hline oaire:version & VoR \\
\hline URL & https://hdl. handle. net/11094/2980 \\
\hline rights & \\
\hline Note & \\
\hline
\end{tabular}

Osaka University Knowledge Archive : OUKA

https://ir. Library. osaka-u. ac. jp/

Osaka University 


\title{
Fast Ion Generation in Ultra Intense Laser Interactions with Plasmas
}

\author{
H.Habara, R.Kodama, Y.Sentoku, Y.Kitagawa, ${ }^{a}$ K.A.Tanaka, K.Mima, and T.Yamanaka \\ Institute of Laser Engineering, Osaka Univ., 2-6 Yamadaoka, Suita, Osaka 565-0871, Japan \\ aFacillity of Engineering, Osaka Univ., 2-6 Yamadaoka, Suita, Osaka 565-0871, Japan
}

\begin{abstract}
In order to study the ion acceleration in ultra-intense laser plasma interactions, we measured neutron spectra from the D-D ion nuclear reactions in the target. 50-100TW / 0.5-1 ps laser light irradiated obliquely deuterated plastic solid targets with different laser polarizations and intensities. Comparing the neutron spectra with the 3-D Monte-carlo simulation indicates ion acceleration in the rear target normal direction at any polarization and increases in the ion energy with laser intensity.
\end{abstract}

Keywords: Ultra intense laser interaction, Ion acceleration, Fast Ignitor, Neutron spectroscopy, beam fusion

\section{INTRODUCTION}

Recent progress of a short pulse high peak power laser development using a Chirp-Pulse Amplification (CPA) method have opened new phases in the field of a laser-plasma interactions. The laser focal intensity is realized to be over $10^{18} \mathrm{~W} / \mathrm{cm}^{2}$ and will be reached at $>10^{21} \mathrm{~W} / \mathrm{cm}^{2}$. Under the intensity, new phenomena in the laser-plasma interaction has been observed such as high order harmonic generation, fast electrons and ions generation and so on $^{2}$. Especially, energetic ions with energies of over $\mathrm{MeV}$ ranges have been concemed from a viewpoint of a laser acceleration, and fast igniter concept ${ }^{1}{ }^{1}$ in Inertial Conferment Fusion.

It is very important to investigate the spatial distribution of accelerated ions for understanding of the acceleration mechanism. To measure the distribution in the target inside, we utilized multi channel spectroscopy of neutrons generated through nuclear reactions. We also developed 3D monte-carlo simulation of ion motion in a solid target to fit the experimental results for in evaluation of the ion spatial distribution and its energy. As one of methods to measure the ion momentum distribution, there is direct observation of the ion at out of the target with track detectors such as a CR-39. However, ion motion can be affected by a strong electric and/or magnetic fields in the plasmas. On the other hand, neutron spectroscopy have an advantage on the investigation of ion momentum at the target inside. Any target potential and magnetic fields can not affect the motion of neutrons generated in the target. Unfortnaly neutron spectra for one direction give us no these dimensional ion momentum. Then we have measured the neutron spectra from three different directions to obtain the ion momentum distribution in the target.

In the second section, we show the three-dimensinal Monte-Carlo simulation to fit the neutron spectra. The third section mentions about experiments setup for ion acceleration and an example of fitting the simulation to the experimental spectra. The forth section is experimental results of laser polarization and intensity dependencies of the ion acceleration. The last is summery.

\section{3D MONTE-CARLO SIMULATION OF ION MOTION IN THE SOLID TARGET}

We developed 3D Monte-carlo simulation to analyze the neutron spectra for several directions. The neutron 
spectra for the different directions are calculated changing initial momentum distributions of the accelerated ions to be fitted to the experimental spectra. We assured that the laser-plasma interaction region is only at the target surface (near skin length) and ions move in solid. The ion motion is affected by elastic and inelastic scattering in the solid target. The ion energy is decreased through elastic scattering by coulomb forces. The stopping power can be written from a Jackson's equation ${ }^{3}$ by

$$
\frac{\partial E}{\partial z}=\frac{\omega_{p}^{2}\left(Z_{e f}\right)^{2} e^{2}}{c^{2} \beta^{2}} G\left(y_{e}\right) \ln \Lambda
$$

where $E$ is the ion energy, $\omega_{p}$ the plasma frequency, $Z_{\text {eff }}$ the effective charge number, $G\left(y_{e}\right)$ the error function, $y_{e}$ the accelerated ion energy divided by background electron or ion temperatures and $\ln \Lambda$ the coulomb logarithm.

On the other hand, the accelerated ions impacts to the ions in the solid target and generate neutrons through nuclear fusion reaction. We used deuteron-deuteron reaction $\left(D[D, n]^{3} \mathrm{He}\right)$ as the neutron generated reaction of which cross sections are well known till sub keV. The DD reaction cross section was refereed from ECPL ${ }^{4}$. Neutron yield is represented by

$$
Y_{n}=n_{a i} n_{t i}\langle\sigma v\rangle d t \quad
$$

where the $\mathrm{n}_{\mathrm{ai}}$ is accelerated ion number density, $\mathrm{n}_{\mathrm{ti}}$ the target ion number density, $\sigma$ the cross section of nuclear reaction, $v$ the relative velocity between accelerated and target ions and $d t$ the reaction time.

The direction of neutrons to the angle of the incident deuteron in the Lab. system can be relatively written by

$$
E_{n}=\frac{-(d) \pm \sqrt{(d)^{2}+4(c)(a)}}{2(c)}
$$

where

$$
\begin{aligned}
& (a)=2 m_{D} c^{2} E_{D}-2 m_{{ }^{H e}} c^{2}\left(Q+E_{D}\right)-Q^{2}-2 Q E_{D}, \\
& (b)=2\left(m_{n} c^{2}+m{ }{ }_{H e} c^{2}+Q+E_{D}\right),
\end{aligned}
$$$$
(c)=4 \cos ^{2} \theta \cdot\left(2 m_{D} c^{2} E_{D}+E_{D}^{2}\right)-(b)^{2} \text {, }
$$

and

$$
(d)=8 \cos ^{2} \theta \cdot m_{n} c^{2}\left(2 m_{D} c^{2} E_{D}+E_{D}^{2}\right)^{2}-2(a)(b) \text {. }
$$

$m_{D}, m_{3} \mathrm{He}$ and $m_{n}$ are the rest mass of deuteron, ${ }^{3} \mathrm{He}$ and neutron, respectively. $\mathrm{Q}$ is the released energy through the nuclear reaction, $\theta$ the incident angle and $\mathrm{E}_{\mathrm{D}}$ the incident energy of the accelerated deuteron.

In the three-dimensional space, we change shapes of an ion momentum distributions in respective coordinates, i.e. $\mathrm{Px}, \mathrm{Py}$ and $\mathrm{Pz}$, where $\mathrm{Px}$ corresponding to the target rear side normal, Py the $y$ axis parallel to the electrical field of the laser light and $\mathrm{Pz}$ the $\mathrm{z}$ axis perpendicular to the electrical field. As examples, we show five different distributions; (a) Isotropic momentum distribution of accelerated ions. (a)-i is a momentum distribution with a Maxwellian energy distribution. The arrow in the target shows the direction of laser incidence. The gray area represents target inside and the white is the vacuum region. (a)-ii and (a)-iii shows the calculated spectra at 180 degree and 90 degree from the target normal direction. (b) Isotropic momentum distribution of accelerated ion with a monochromatic energy. Other figures conditions are shown as a same as (a). (c) Momentum distribution collimated the target normal direction and the calculated spectra with Maxwellian energy distribution. (d) Momentum distribution and calculated spectra collimated 45 deg. from the target normal with a Maxwellian energy distribution. (e) Compricated momentum distribution shape and its calculated spectra with a Maxwellian energy distribution. Even if there is a similar shape spectrum, neutron spectra with multi channel detection can identify the ion momentum distribuion. 

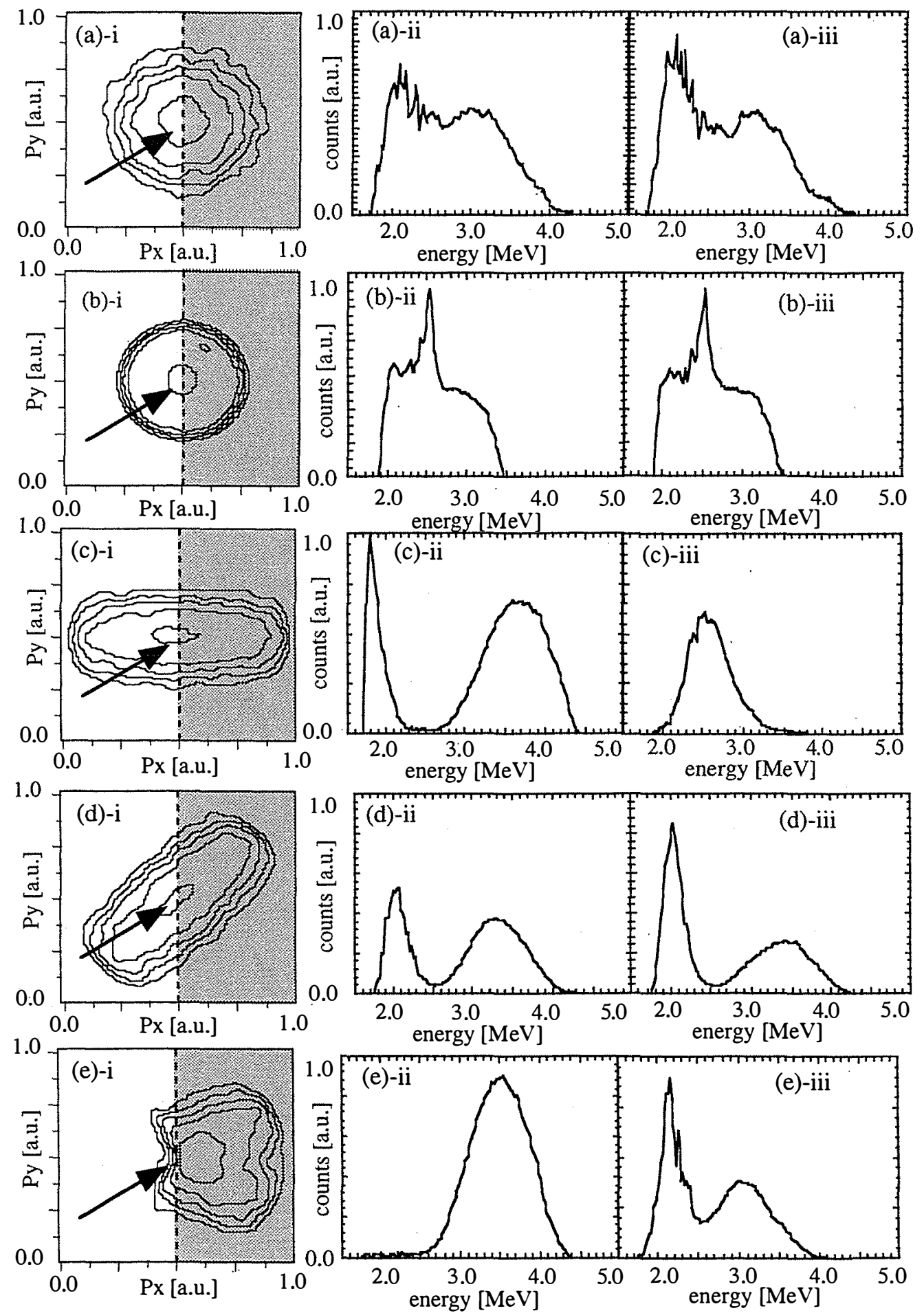

Fig. 1 Examples of (i) ion momentum distributions and (ii), (iii) calculated spectra from 180 deg. and 90 deg. to the target normal. The arrow shows the direction of the laser incidence. The gray area represents target inside and the white is a vacuum region. (a)Isotropic distribution with a Maxwellian energy distribution. (b) Isotropic distribution with a monochromatic energy ions. (c) Momentum distribution collimated to the target normal direction with a Maxwellian energy distribution. (d) Momentum distribution collimated to the 45 degree to the target normal with a Maxwellian energy distribuion. (e) Compricated momentum distribution. 


\section{EXPERIMENTS}

\subsection{Experimental Setup}

The experiments were performed by using 50TW short pulse glass laser systems at Institute of Laser Engineering(ILE), Osaka University. The $1.05 \mu \mathrm{m}$ light laser energies were 20-50J on targets and the pulse widths were 450-800fs from a second-order autocorrelator. The pulse shape was identified with correlation between the autocorrelator signals and spectra from a spectrometer as well as the result from a third-order autocorrelator. The prepulse level was monitored with a pin diode to be less than $10^{-4}$ to the main pulse. A deuterated plastic (CD) target was irradiated by the short pulse laser light through $\mathrm{f} / 3.8$ focusing mirror. The intensity of the main pulse on the target was $10^{19} \mathrm{~W} / \mathrm{cm}^{2}$ from the spot size $(\sim 15 \mu \mathrm{m})$ obtained with far field pattern of the laser and an $\mathrm{x}$-ray pinhole camera.

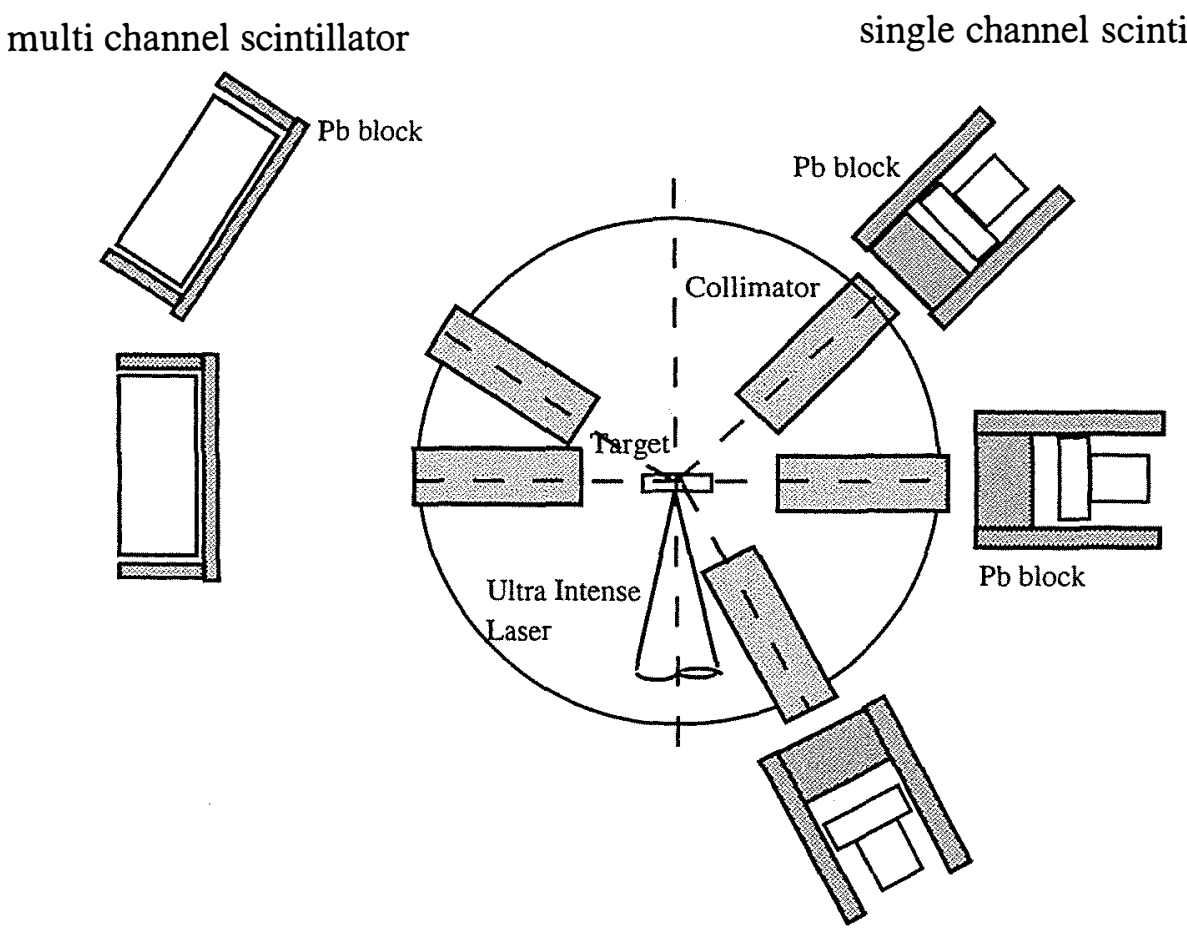

Fig.2 Experimental setup of the scintillators. Typical we use two or three scintillators all of this. To reduce a gamma noise from laser-mater interactions, lead block is placed in front and side of the scintillators and collimator was placed before the scintillators. 
Neutron spectra was detected with a time of light (TOF) method using neutron spectrometers consisted of plastic scintillators for different angles. Two different types of the spectrometers were used in the experiment: one type was a single channel detector system to obtain the spectra form the temporal current profile of the scintillator signals and another a multi-channel detector system to obtain the spectra from the channel event number 5 . Reliable detection ranges of neutron energies of the system were under $20 \mathrm{MeV}$. Energy resolutions of neutron spectra were about $29 \mathrm{keV}$ (multi-channel detector) and $110 \mathrm{keV}$ (single channel detector). The two or three neutron spectrometers were set up from different view angles.

Figure 2 shows the experimental setup. All the neutron detectors had $5 \mathrm{~cm}$-thick cylindrical polyethylene collimators with $5 \mathrm{~mm}$-thick lead blocks in the chamber to reduce scattered electrons and a gamma-ray noise from the chamber inside, e.g. from the chamber wall and the other diagnostics such as pinhole cameras. In order to reduce the signal level of a gamma-ray noise from the target to the neutron signals, $15 \mathrm{~cm} \mathrm{~Pb}$ blocks were placed at the front of the scintillators. $5 \mathrm{~cm}$ thick lead blocks were also set at the side of the detector to protect it from gamma rays. Neutron scatter effects on the spectra due to the lead block were calibrated with thermal neutrons created by GMB implosion, resulting in the negligible effect of scattering neutrons in the spectra.

\subsection{Neutron spectra}

We show an examples of neutron spectra. The $1 \mu \mathrm{m}$ laser light was obliquely irradiated a CD $5 \mu \mathrm{m}$ target with mixed polarization (18 degree of $s$ - polarization and 38 degree of $p$-polarization) at intensities $10^{19} \mathrm{~W} / \mathrm{cm}^{2}$. Solid lines in all figure show the observed neutron spectra at a laser incident angle of 40 degree to the target normal for the $s$-polarization condition. Figure 3(a) shows experimental spectra at three observation angles: 90 deg.(solid line), 56 deg.(dashed line) and 38 deg.(dotted line) to the target normal, relatively. The signal level on the figure is normalized to be a neutron counts per solid angles taking account of the detector sensitivity. The peak of the spectra for the target front side shifts to the lower energy side $\left(2.3 \mathrm{MeV}\right.$ at $39^{\circ}, 2.1 \mathrm{MeV}$ at $\left.56^{\circ}\right)$ from $2.45 \mathrm{MeV}$ given by the D-D thermal fusion reaction and there is no shifts in the spectra at the target side(dashed and dotted line). These shifts suggest that ions are accelerated into target inside taking account of the momentum conservation, which is similar to a Doppler effect on the neutron spectra. We performed 3D Monte-carlo simulation to fit the experimental results. The calculated neutron spectra from the ion distribution indicates the collimation of ions to rear target normal.

Three-dimensional momentum spatial distributions collimated the target rear direction are given as a momentum ratio of $\mathrm{Px}: \mathrm{Py}: \mathrm{Pz}=2.3: 1: 1$. The two dimensional distribution on the $\mathrm{x}-\mathrm{y}$ plane is shown as a contour plot in Fig.3(c). Each lines represents $10^{0.2}$ of the ion number in a logarithmic. The ion numbers on the $x-y$ plane are integrated in $\mathrm{z}$ coordinate. It is clear that the ions are accelerated in the direction of rear target normal. The ion energy to the $\mathrm{x}$-direction corresponding to the rear target normal is $330 \mathrm{keV}$ whereas the energy to the $\mathrm{y}$ and $\mathrm{z}$-direction is about $70 \mathrm{keV}$. 


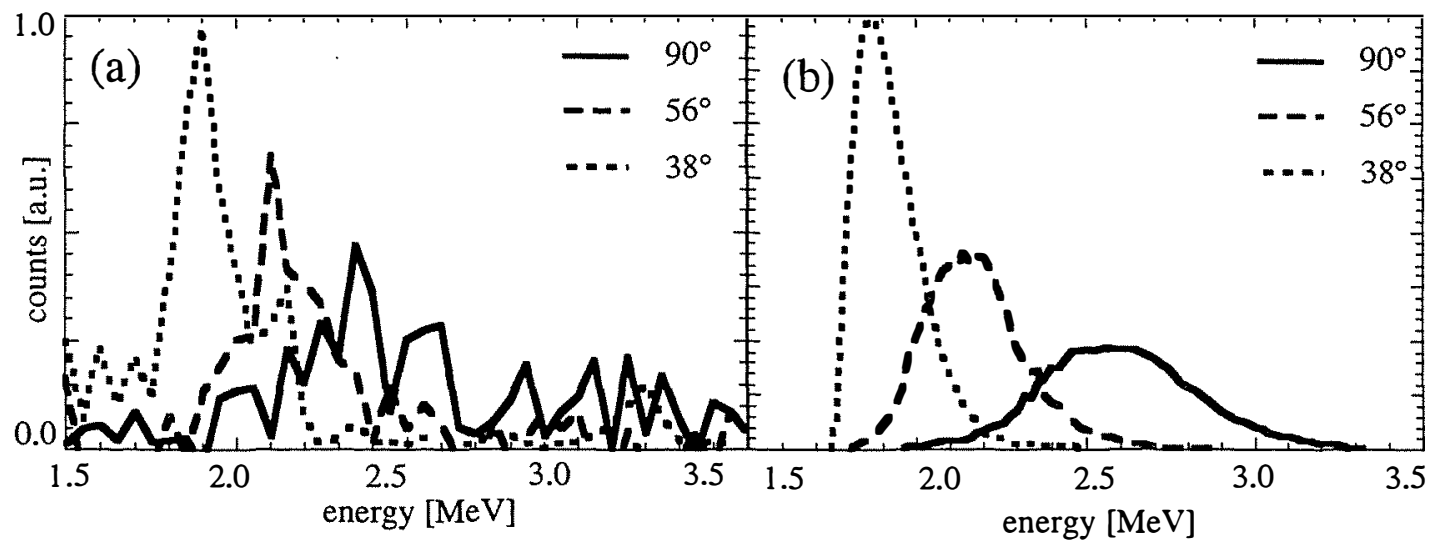

target surface

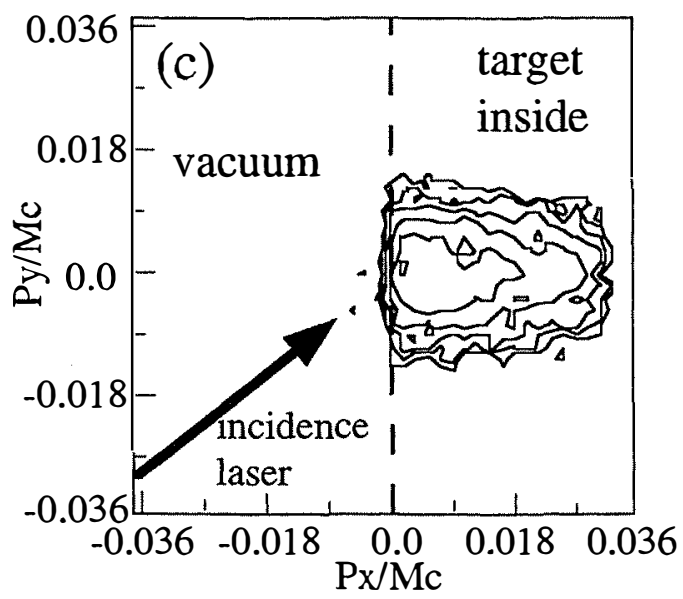

Fig. 3 Neutron spectra and for ion momentum distribution. (a) Experimental spectra. Solid line shows the spectra for 90 deg. to the target normal and dashed and dotted lines are for 56 deg. and 38 deg., respectively. (b) The calculated neutron spectra to fit the experimental result. Solid line, dashed line and dotted line are the spectra for $90 \mathrm{deg}$., $56 \mathrm{deg}$. and 38 deg., respectively. (c) Two dimensional momentum distribution on the $x-y$ plane as shown in conture plots. Each lines represents $10^{0.2}$ of the ion number in a logarithmic.

\section{ION ACCELERATION MECHANISM}

\subsection{Polarization dependence}

We obtained the laser polarization dependence of ion acceleration in ultra-intense laser interactions with solid targets. Two different liner polarization conditions, i.e. $s$-polarization and $p$-polarization, were taken to measure the polarization dependence of the neutron spectra. Table 1 shows the summary of the ion momentum distributions and temperature of accelerated ions to the rear target normal from the experimental spectra fitting at different laser polarizations at $10^{19} \mathrm{~W} / \mathrm{cm}^{2}$ without large prepulse $\left(<10^{-4}\right)$. At both the laser conditions, the direction of the ion acceleration was rear target normal. The results shows there is the possibility of the ion acceleration mechanism is static field by a charge separation between electrons and ions ${ }^{6}$. On the other hand, the momentum distribution and the energies of ions were clearly different at different polarizations. The energy of accelerated ions (Maxwellian temperature:330keV) 
to the rear target nonmal is larger than that of the $p$-polarization $(120 \mathrm{keV})$. This difference derived from different mainly laser absorption mechanisms, which are $\mathrm{JxB}$ absorption ${ }^{7}$ at $s$-polarization and strong laser absorption at p-polarization called 'Brunel absorption ${ }^{8,9}$. From these results, we decide ion acceleration mechanism is static field by a charge separation between electrons and ions ${ }^{6}$. We also estimated total neutron yield from the fitting the experimental results, resulting in about 100 times larger yield at $s$-polarization compared with $p$-polarization. These resuls also show higher energy ions are much accelerated at $s$-polarization.

\begin{tabular}{|c|c|c|c|}
\hline & Px:Py:Pz & Ex & $Y_{n}$ \\
\hline s-polarization & $2.3: 1: 1$ & 330 & $10^{\wedge} 8$ \\
\hline p-polarization & $1.4: 1: 1$ & 120 & $10^{\wedge} 6$ \\
\hline
\end{tabular}

Table 1 Summary of momentum distributions and temperature of ions from the experiment spectra fitting by 3D Montecarlo simulation at different polarizations. Px is corresponding to the rear target normal, Py the $y$ axis parallel to the target surface. Ex shows the temperature of accelerated ions along the $x$-direction, plus shows the temperature to the target inside and minis that of the vacuum side. Yn shows neutron yield assuming $4 \pi$ approximation.

\subsection{Intensity dependence}

We obtained the laser intensity dependence of ion acceleration. The laser condition was fixed at $p$-polarization 20 deg. incidence. Figs.5(a) and (b) show neutron spectra from $43^{\circ}$ (solid line) and $87^{\circ}$ (dashed line) to target normal at $2 \times 10^{18} \mathrm{~W} / \mathrm{cm}^{2}$ and $1 \times 10^{19} \mathrm{~W} / \mathrm{cm}^{2}$, respectively. The signal level on the figure is normalized to be a neutron counts per solid angles taking account of the detector sensitivity. The peaks of neutron spectra at $2 \times 10^{18} \mathrm{~W} / \mathrm{cm}^{2}$ are not sifted from 2.45MeV so much. On the other hand, neutron spectra at an intensities of $1 \times 10^{19} \mathrm{~W} / \mathrm{cm}^{2}$ show clear shift of the peak from $2.45 \mathrm{MeV}$ The peak of the spectra for the target front side shifts to the higher energy side $\left(2.9 \mathrm{MeV}\right.$ at $43^{\circ}, 3.0 \mathrm{MeV}$ at $87^{\circ}$ ) from $2.45 \mathrm{MeV}$ given by the D-D thermal fusion reaction. The neutron counts at $1 \times 10^{19} \mathrm{~W} / \mathrm{cm}^{2}$ is about a hundred times larger than the counts at $2 \times 10^{18} \mathrm{~W} / \mathrm{cm}^{2}$. These differences in the peak shifts and neutron of neutron spectrum indicates the acceleration of ions with higher energy at higher laser intensities. Using 3D Monte-carlo code, the energy of accelerated ion is estimated (a) $40 \mathrm{keV}$ and (b) $80 \mathrm{keV}$ assuming ion energy distribution as Maxwellian. Both of accelerated ion number is constant at a few time of $10^{13}$ from the spectral fitting. If the ion is accelerated by the static field, the energy of accelerated ion increase with laser intensity. Therefore the results is consistent with the acceleration mechanism is a static field as shown in previous section.
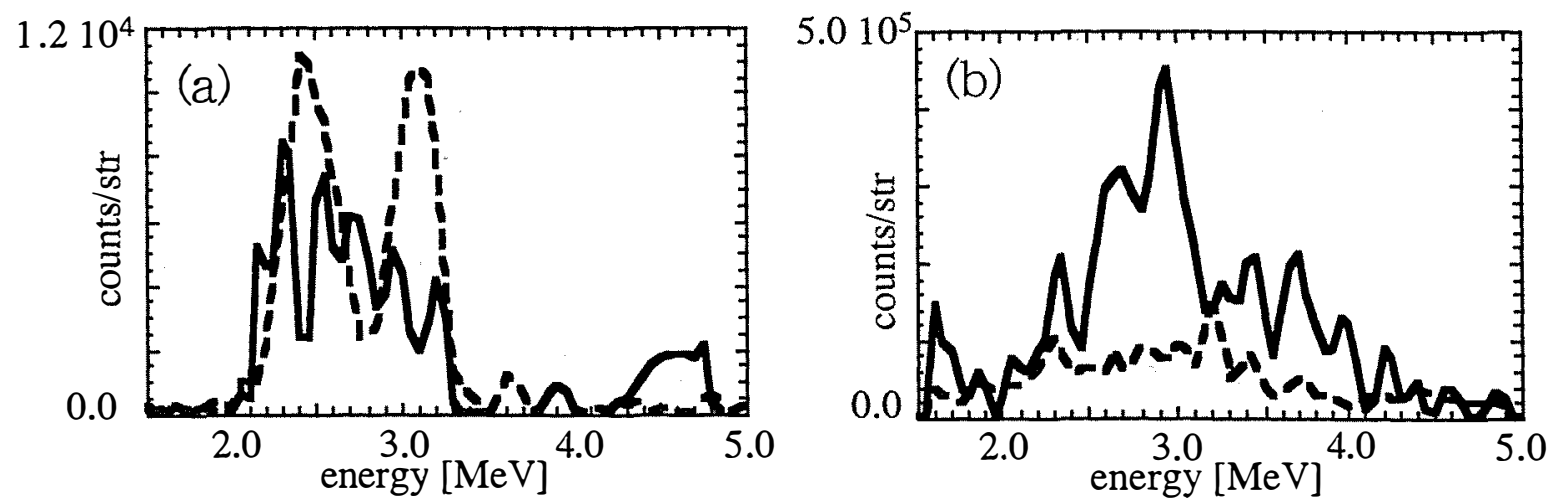

Fig 5 Laser intensity dependence at neutron spectra (a)Neutron spectra at intensites of $10^{18} \mathrm{~W} / \mathrm{cm}^{2}$ at $43^{\circ}$ (solid line) and $87^{\circ}$ (dotted line) from target normal. (b) Neutron spectra at intensites of $10^{19} \mathrm{~W} / \mathrm{cm}^{2}$. The other figure conditions are as same as Fig.5(b). 


\section{Summery}

We measured laser polarization and intensity dependence of neutron spectra to study the ion acceleration mechanism in ultra-intense laser interactions with solid targets. Comparing the neutron spectra with the 3-D Monte-carlo simulation indicates ion acceleration in the rear target normal direction at any polarization. And the ion energy increased in the laser intensity. These results is consistent that the ion acceleration with a static electric field by charge separation between ions and electrons.

\section{ACKNOWLEDGE}

We acknowledge all the technical supports of the engineering at Institute of Laser Engineering for the laser operator, target fabrication and data acquisition. We specially thank Dr. T.Norimatu, Dr. Fujita, Dr. Yoshida, K.Sawai, T.Kawasaki, T.Matsuo, K.Suzuki, Y.Kimura, O.Maekawa,and T.Komenou.

\section{REFERENCES}

1. M.Tabak, J.Hammer, M.E.Glinsky, W.L.Kruer and S.C.Wilks, "Ignition and high gain with ultrapowerful lasers", Phys. Plasmas 1, 1626 (1994).

2. A.P.Fews, P.A.Norreys, F.N.Beg, A.R>Bell, A.E.Dangor, C.N.Danson, P.Lee and S.J.Rose, "Plasma Ion Emission from High Intensity Picosecond Laser Pulse Interactions with Solid Targets", Phys. Rev. Lett. 73, 1801 (1994).

3. J.D.Jackson, "Classical Electrodynamics 2nd. Edition.", chapter 13 (John Wiley \& Sons.Inc. , N.Y1975).

4. R.J.Howerton, R.E.Dye, M.H.MacGregor, S.T.Perkins, "Index to the LLNL Evaluated Charged-Particle Library", (Lawrence Livermore National Laboratory. 1986)

5. N.Izumi, K.Yamaguchi, T.Yamagajo, T.Nakano, T.Kasai, T.Urano, H.Azechi, S.Nakai and T.Iida, "A highly efficient neutron time-of-flight detector for inertial confinement fusion experiments", Rev. Scientific Instruments 70, pp 1221-1224 (1999).

6. H.Habara, R.Kodama, Y.Sentoku, N.Izumi, Y.Kitagawa, K.A.Tanaka, K.Mima and T.Yamanaka, "Study of Ion Acceleration in Ultra-Intense Laser-Plasma Interactions with Neutron Spectroscopy", ILE Internal Report, ILE9905FE.

7. E.G.Gamaly, Phys. Fluids 5, 3765 (1993).

8. $\quad$ F.Brunel, "Not-So-Resonant, Resonance Absorption", Phys. Rev. Lett. 59, 52 (1987).

9. P.Gibbon and A.R.Bell, "Collisionless Absorption in Sharp-Edged Plasmas", Phys. Rev. Lett. 68, 1535 (1992). 